\title{
One-stage simultaneous hip-preserving surgeries for the management of bilateral femoral head osteonecrosis: a mean 7.0- year follow-up
}

Wenjun Feng ${ }^{1 *+}$, Pengcheng $\mathrm{Ye}^{1+}$, Shihao $\mathrm{Ni}^{2+}$, Peng Deng ${ }^{2}$, Lu Lu ${ }^{3}$, Jinlun Chen ${ }^{1}$, Jianchun Zeng ${ }^{1}$, Xinyu $\mathrm{Qi}^{2}$, Jie $\mathrm{Li}^{1}$, Ke Jie ${ }^{4}$, Houran $\mathrm{CaO}^{2}$, Zhijun Yue ${ }^{2}$, Haitao Zhang ${ }^{2}$ and Yirong Zeng ${ }^{1 *}$

\begin{abstract}
Background: A retrospective study was conducted to evaluate and compare the clinical and radiological outcomes of one-stage fibular impaction allografting and vascularized greater trochanter flap autografting for the treatment of bilateral osteonecrosis of the femoral head (ONFH).

Methods: Patients who underwent one-stage aforementioned hip-preserving surgeries due to bilateral ONFH were retrospectively reviewed from January 2008 to December 2013. Sixty-nine patients (138 hips) with a mean age of 31.5 years and mean follow-up of 7.0 years were included. Hips that underwent fibular impaction allografting and vascularized greater trochanter flap autografting were assigned as group A and group B, respectively. Harris Hip Score (HHS) and Visual Analogue Scale (VAS) were used for clinical evaluation, and a series of X-ray images were used for radiological assessment. For inter-group analysis, the paired $t$ test was used for continuous data, and the Wilcoxon rank sum test was used for non-parametric data, while the Mann-Whitney $U$ test was used for intra-group analysis.
\end{abstract}

Results: The HHS and VAS in both groups A and B had a substantial advancement when compared with the preoperative level $(p<0.01)$. Fibular impaction allografting can achieve more pain relief $(p<0.01)$, though no clinical difference was found in terms of minimal clinically important difference (MCID $<10$ points). Group A showed better radiological results than group $\mathrm{B}(p=0.04)$. It was discovered that the appropriate indication for each procedure was patients with Association for Research on Osseous Circulation (ARCO) stages II and III, respectively.

Conclusion: One-stage hip-preserving surgeries for the management of bilateral ONFH could obtain good medium and long-term outcomes. It was recommended that fibular impaction allografting is more suitable for patients in ARCO stage II, while for patients in ARCO stage III, vascularized greater trochanter flap autografting is a better preference.

Trial registration: Retrospectively registered.

Keywords: Osteonecrosis of the femoral head, Hip-preserving, Fibular impaction allografting, Vascularized greater trochanter bone flap, Comparative study

\footnotetext{
*Correspondence: foreverarsene@126.cm; 13711456292@163.com

tWenjun Feng, Pengcheng Ye and Shihao Ni contributed equally to this

work.

${ }^{1}$ The First Affiliated Hospital of Guangzhou University of Chinese Medicine,

Jichang Road 16\#, District Baiyun, Guangzhou, Guangdong, China

Full list of author information is available at the end of the article
}

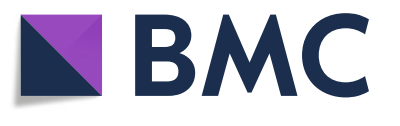

(c) The Author(s). 2019 Open Access This article is distributed under the terms of the Creative Commons Attribution 4.0 International License (http://creativecommons.org/licenses/by/4.0/), which permits unrestricted use, distribution, and reproduction in any medium, provided you give appropriate credit to the original author(s) and the source, provide a link to the Creative Commons license, and indicate if changes were made. The Creative Commons Public Domain Dedication waiver (http://creativecommons.org/publicdomain/zero/1.0/) applies to the data made available in this article, unless otherwise stated. 


\section{Introduction}

Osteonecrosis of the femoral head (ONFH) mainly affects young individuals in their thirties and forties [1]. Although multiple risk factors have been identified [2], such as alcohol intake, corticosteroid abuse, and trauma, the underlying pathophysiology has still not been wellrecognized, which makes its treatment very challenging. The terminal course of ONFH is femoral head collapse, articular cartilage degeneration, and subsequent latestage osteoarthritis when no timely treatment initiated $[3,4]$. Total hip arthroplasty (THA) can resolve the condition with definite success and long-term satisfactory outcomes [5]. However, regardless of improvements in joint prosthesis design, instruments, and surgical techniques, THA is unlikely to be endured for the rest of the life of the acceptors, especially for young individuals. Thus, it is of great significance to preserve the necrotic femoral head, especially for young patients.

A lot of efforts have been made from conservative methods to surgical interventions for the management of ONFH. For those with early-stage asymptomatic femoral head necrosis, nonsurgical treatments might be effective, such as administration of antithrombotic drugs, bisphosphonates, lipid-lowering agents, and some herbals [6-9]. Non-weight-bearing of the affected hip with the assistance of crutches and physiotherapy was also reported to be useful [10]. Hip-preserving surgeries should be always in our priority when indexed indications are met. Various hip-preserving surgeries have also been investigated for those symptomatic large lesion pre-osteoarthritis cases, such as non-vascularized [11] or vascularized bone grafting [12], fibular impaction grafting [13], core decompression [14], rotational osteotomy [15], and stem cells implantation [16]. In brief, the ultimate goals for the treatment of ONFH are to maintain hip function, no pain, and delay or avoid THA conversion.

Previous studies have described that ONFH often occurred in bilateral hips and the incidence was as high as $78 \%[17,18]$. And it is commonly seen that those two hips are in different stages. For bilateral ONFH, Marcus et al. [19] reported that one-sided Phemister-type bone grafting for patients with Association for Research on Osseous Circulation (ARCO) I and II stages and concurrent THA for ARCO IV stage patients can achieve 90\% success. Lih-Yuann et al. [20] who performed one-stage fibular grafting and THA for 36 patients concluded that patients obtain more benefits because of cost-effective and less perioperative morbidity and that the headpreserved hips had better survivorship. Zeng et al. [13] reported similar findings in 18 patients with both affected hips, in which one side underwent THA and the other hip accepted fibular grafting at one-stage. However, to our knowledge, one-stage hip-preserving surgery for both hips has been rarely reported in currently published articles. Cao et al. [21] who conducted a randomized clinical trial to compare free vascularized fibular grafting with core decompression in bilateral ONFH management found that both techniques can obtain good mid-term outcomes and the survivorship was $90.5 \%$ and $95.2 \%$, respectively. However, there was no statistical significance between those two techniques. Besides, the enrolled cases were mostly pre-collapse asymptomatic cases, and mean follow-up was only 2 years. Thus, we conducted a retrospective study to evaluate and compare the clinical and radiological outcomes of one-stage fibular impaction allografting and vascularized greater trochanter flap autografting for the treatment of bilateral ONFH. Neither of those two procedures is a new technique. However, to our knowledge, no prior studies have been published. We asked the following questions: (1) What is the effect of one-stage fibular impaction allografting and vascularized bone flap autografting for the treatment of bilateral ONFH? (2) Is one procedure superior to the other one from the improvement of the Harris Hip Score (HHS) and Visual Analogue Scale (VAS)? (3) What is the appropriate indication for each procedure?

\section{Materials and methods}

It was a monocentric and retrospective cohort study. Patients who underwent one-stage fibular impaction allografting and vascularized greater trochanter flap autografting due to bilateral ONFH from January 2008 to December 2013 were included. All surgeries were performed by one single experienced surgeon. The participants were all in ARCO stages II and III. All patients were informed that the data might be used for further study, and written consent was obtained preoperatively. This study was approved by the Ethics and Academy Committee of the First Affiliated Hospital of Guangzhou University of Traditional Chinese Medicine (Grant number [2017]124), and it complied with the Declaration of Helsinki. Patients diagnosed with bilateral ONFH at the age between 18 years and 45 years were included. The excluded criteria were previous surgical intervention of the affected hip, femoral neck and head fracture, hip infections, tumor, musculoskeletal disorders, hematologic diseases, unable to understand and follow postoperative instructions, and mental health deficiency.

We recruited 69 patients (138 hips) who underwent one-stage abovementioned hip-preserving surgeries for bilateral ONFH management. The fibular impaction allografting hips were assigned into group $\mathrm{A}$, while the vascularized bone flap autografting hips were allocated to group B. Patients were contacted through regular revisit in the out-patient department to complete postoperative follow-up. And no patient was lost follow-up. 
The latest follow-up time was in May 2018, and the endpoint of hip-preserving surgeries was determined by the time of THA conversion. Data collection consisted of age, gender, height, weight, body mass index (BMI), etiology, follow-up time, operation time, blood loss, risk factors, and baseline ARCO stages. The details of the demographics were presented in Table 1 and Table 2.

\section{Surgical procedures}

The procedures of fibular impaction allografting and vascularized greater trochanter flap autografting both had been described in previous studies $[12,13]$. In brief, for hips in group A, a $5-\mathrm{cm}$ to $6-\mathrm{cm}$ incision originated from the tip of the greater trochanter to the femur distally was used. A $2.5-\mathrm{mm}$ Kirschner pin was inserted directed toward the necrotic area until $0.5 \mathrm{~cm}$ below the subchondral bone under X-ray fluoroscopy. A customized hollow reamer was used to broach a tunnel via the positioning Kirschner pin, and a metal alloy $\mathrm{T}$-shaped hand driller with different diameters and directions was used for necrotic bone complete debridement. Commercial allogeneic cancellous bone granules with a median size of $5 \mathrm{~mm}$ were then tightly impacted, followed by allogeneic fibular implantation through the tunnel, while for hips in group B, a modified Smith-Peterson approach with an incision of $15 \mathrm{~cm}$ to $20 \mathrm{~cm}$ in length was used. Firstly, the greater trochanter with the lateral femoral circumflex artery transverse branch was isolated and protected in cases of vascularity damage. Secondly, a window-like approach on the collapsed cartilage surface accessed to the necrotic area was made to remove all necrotic bone and tissues, and harden boundary was penetrated with $2.5-\mathrm{mm}$ Kirschner pin for the purpose of blood supply provision from the unaffected region. Thirdly, the free iliac bone flap was harvested from the outer lip of the iliac crest and filled into the cavity after trimming in a matched shape. Fourthly, the predisposed vascularized greater trochanter was implanted through a

Table 1 Baseline demographic characteristics of the enrolled participants

\begin{tabular}{ll}
\hline Characteristics & 69 participants (138 hips) \\
\hline Age (years; mean \pm SD [range]) & $31.5 \pm 7.5(21-45)$ \\
Gender (male/female) & $30 / 39$ \\
Height (cm; mean \pm SD [range]) & $165.4 \pm 6.9(155-178)$ \\
Weight (kg; mean \pm SD [range]) & $62.0 \pm 9.2(58-80)$ \\
BMI (mean \pm SD [range]) & $22.7 \pm 2.2(18.8-26.0)$ \\
Follow-up (years; mean \pm SD [range]) & $7.0 \pm 1.0(5-10)$ \\
Risk factors & \\
$\quad$ Idiopathic & 14 \\
$\quad$ Corticosteroid & 30 \\
$\quad$ Alcohol & 25 \\
\hline
\end{tabular}

groove along the femoral neck without entrapment and stretch, and lift-up cartilage surface was fixed and reshaped in more congruent morphology; the femoral head was then reduced. Patients were not allowed for weight-bearing at the first 6 weeks postoperatively, followed by gradually partial weight-bearing depending on the necrotic bone repair process, and total weightbearing was permitted only after 6 months postoperatively. Tibial tuberosity skeletal traction was performed in the vascularized bone flap autografting hips immediately after surgery and lasted for 6 weeks, and then suffered limb skin traction was subsequently continued until 6 months postoperatively, while for the fibular impaction allografting hips, the skin traction sustained during the whole recovery period.

\section{Outcome evaluation}

The clinical and radiological assessments were done by two independent researchers. Patients were examined at preoperative and postoperative 3, 6, and 12 months and 1 year annually thereafter. HHS and VAS were used for clinical outcome evaluation. Although HHS system is used for evaluating hip function, it is extremely difficult to distinguish the function disability from one hip to the contralateral hip when considering both hips were onestage surgically treated in one single patient, because daily activities such as sitting, climbing stairs, putting on socks and shoes, using of public transportation, and walking ability are completed by both affected hips. Jasvinder et al. [22] reported that minimum clinically important difference (MCID) showed a reliable predictive ability of the HHS questionnaire, and defined a threshold of 16 to 18 points. However, the participants in our study were inter-group difference comparison for one same patient; we defined a MCID of 10 points on the 100-point HHS as Cao et al. [21] reported. X-ray images of anteroposterior and frog lateral view at preoperative and each postoperative were obtained for radiological evaluation in terms of femoral head collapse and necrotic region repair process. It was regarded as improved when femoral head morphology is stable with necrotic region partial or complete repair; stable was defined as no or subtle femoral head collapse and no increased necrotic region; aggravated was determined when progressive femoral head collapse, enlarged necrotic region, and presence of narrow joint space and osteoarthritis were seen. A survivor analysis was also performed. It was considered a clinical failure when subsequent THA conversion was indicated because of deteriorating pain, hip dysfunction, progressive femoral head collapse, and late-stage osteoarthritis. Postoperative complications were also recorded. 
Table 2 Baseline ARCO stage, operation time, and blood loss in groups A and B

\begin{tabular}{llll}
\hline Parameters & Group A & Group B & \\
\hline ARCO stage & 12 & 3 & \\
IIA & 28 & 5 & \\
IIB & 19 & 5 & \\
IIC & 4 & 18 \\
IIIA & 4 & 18 & \\
IIIB & 2 & 20 & \\
IIIC & $50.5 \pm 6.5(41-60)$ & $170.6 \pm 7.8(161-180)$ & $180.7 \pm 12.5(165-200)$
\end{tabular}

$A R C O$, Association for Research on Osseous Circulation. ${ }^{*} p$ values indicated inter-group differences comparison. Continuous data was analyzed via paired $t$ test, while non-parametric data was analyzed via the Wilcoxon rank sum test

\section{Statistical analysis}

For quantitative continuous data, it was expressed as means $( \pm S D)$, while qualitative variables were summarized as count and percentage. For inter-group analysis, the paired $t$ test was used for continuous data, and the Wilcoxon rank sum test was used for non-parametric data, while the Mann-Whitney $U$ test was used for intragroup analysis. All analyses were performed using SPSS statistical software (version 18.0, IBM Corporation, USA). $p$ values less than 0.05 were considered to be statistical significance.

\section{Results}

The HHS and VAS in groups A and B improved from preoperative $70.7 \pm 3.5$ points (range, 64 to 76 points), $58.1 \pm 5.0$ points (range, 48 to 70 points), $4.8 \pm 1.2$ points (range, 3 to 9 points), and $8.0 \pm 1.9$ points (range, 4 to 10 points) to postoperative $92.4 \pm 4.0$ points (range, 80 to 98 points), $84.2 \pm 5.8$ points (range, 70 to 96 points) points, $1.1 \pm 1.0$ points (range, 0 to 4 points), and $2.5 \pm$ 1.9 points (range, 0 to 6 points), respectively (Table 3 ). It revealed that postoperative HHS and VAS in both groups had a substantial advancement when compared with the preoperative level $(p<0.01)$. MCID (the mean HHS difference between groups $\mathrm{A}$ and $\mathrm{B}$ at the latest follow-up) was 8.2 points (95\% confidential interval (CI), 2.4 to 23.2 points). It suggested that there was no clinical significance between these two groups, because MCID did not exceed a priori of 10 points thresholds. The delta

Table 3 Preoperative and the latest follow-up HHS and VAS in groups A and B

\begin{tabular}{lllll}
\hline Parameters & Group A & $p$ value* & Group B & $p$ value $^{*}$ \\
\hline Pre-HHS (mean \pm SD) & $70.7 \pm 3.5$ & $<0.01$ & $58.1 \pm 5.0$ & $<0.01$ \\
Post-HHS (mean \pm SD) & $92.4 \pm 4.0$ & & $84.2 \pm 5.8$ & \\
Pre-VAS (mean \pm SD) & $4.8 \pm 1.2$ & $<0.01$ & $8.0 \pm 1.9$ & $<0.01$ \\
Post-VAS (mean \pm SD) & $1.1 \pm 1.0$ & & $2.5 \pm 1.9$ &
\end{tabular}

HHS, Harris Hip Score; VAS, Visual Analogue Scale. * $p$ values indicated intragroup difference comparison, and the Mann-Whitney $U$ test was used
VAS (the last follow-up postoperative scores minus preoperative scores) in group A was - 3.7 points (95\% CI, 4.9 to -2.5 points), while in group B it was -5.8 points (95\% CI, -7.6 to -3.5 points); hips in group B suggested more pain alleviation $(p<0.01)$. The operation time in group $B$ is much more than that in group $A$, which was $50.5 \pm 6.6 \mathrm{~min}$ (range, 41 to $60 \mathrm{~min}$ ) and $170.6 \pm 7.8 \mathrm{~min}$ (range, 161 to $180 \mathrm{~min}$ ), respectively $(p<0.01)$.

The ARCO classification system was used for the qualitative analysis of femoral head collapse and necrotic repair process. According to abovementioned assessing criterion, 48 hips had improved results, 16 hips had stabled results, and 5 hips had aggravated results in the group A, while in the group B, there were 22 hips, 22 hips, and 25 hips in each turnover outcomes, respectively (Fig. 1). Wilcoxon test revealed that there was clinical significance between these two groups and group A showed better results $(p=0.04)$.

Three hips (all were in ARCO stage IIIB) in group A need a THA conversion a mean postoperative 5.6 years (range, 4 to 7 years). However, 10 hips received THA in group $\mathrm{B}$ at a mean of 6.5 years (range, 5 to 8 years) (Fig. 2). There were 1 hip in ARCO stage IIC, 3 hips in ARCO stage IIIB, and 6 hips in ARCO stage IIIC. There was 1 hip in group $B$ that had to underwent hippreserving revision surgery at postoperative 5 years for the improvement of hip function without pain. The patient was satisfied with the outcomes at the last 10-year follow-up. The survival rates in groups A and B were $95.7 \%(66 / 69)$ and $85.5 \%$ (59/69), respectively. No significance was revealed $(p=0.08)$. A slight negative correlation between the preoperative ARCO stage and the last follow-up clinical outcomes was found (Spearman correlation test, $\rho=-0.19, p=0.04 ; \rho=-0.28, p=0.02$ in groups $\mathrm{A}$ and $\mathrm{B}$, respectively).

We further detected the appropriate indication of each procedure. The delta HHS (the latest follow-up HHS minus preoperative HHS) of groups $\mathrm{A}$ and $\mathrm{B}$ in hips with ARCO stage II (contained IIA, IIB, and IIC) and 


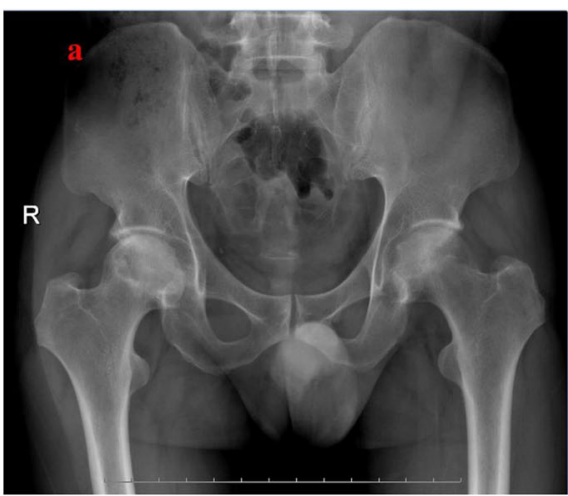

(a)

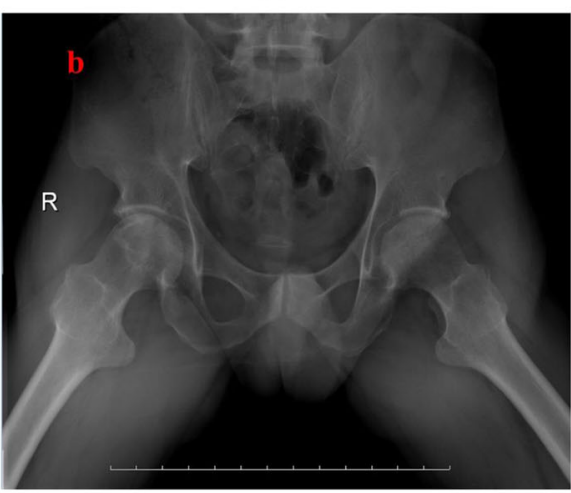

(b)

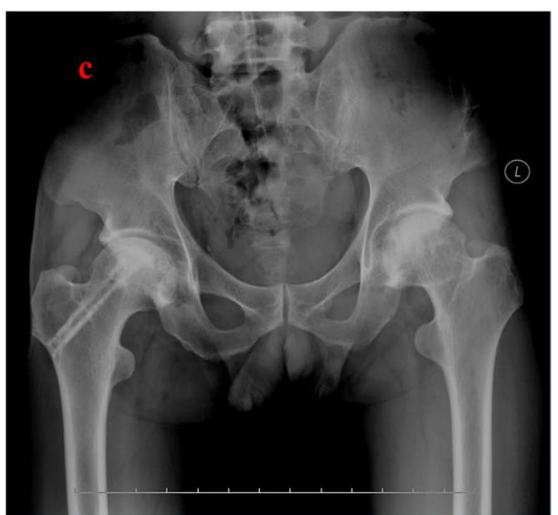

(c)

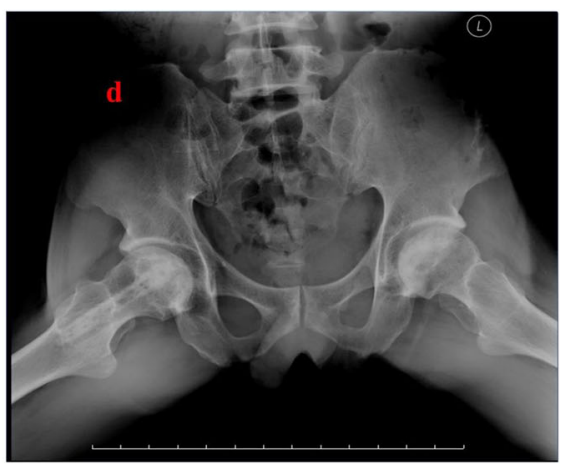

(d)

Fig. 1 a, b 41-year-old male patient complained bilateral hip pain for 2 years. c, d The patient accepted one-stage fibular impaction allografting in the right hip and vascularized greater trochanter flap with the combination of cortico-cancellous iliac bone autografting. X-rays showed completed new bone formation and normal femoral head morphology without the presence of osteoarthritis at postoperative 8-year follow-up

ARCO stage III (IIIA, IIIB, and IIIC) were 26.1 \pm 7.0 points (95\% CI, 11.9 to 36.0 points), $21.2 \pm 4.7$ points (95\% CI, 16.8 to 28.0 points), $24.2 \pm 1.1$ points (95\% CI, 22.5 to 25.3 points), and $27.8 \pm 4.4$ points (95\% CI, 22.9 to 35.0 points). It showed that the appropriate indication for fibular impaction allografting was patients with ARCO stage II $(p<0.01)$, while vascularized greater trochanter flap with the combination of cortico-cancellous iliac bone autografting was the appropriate indication for patients with ARCO stage III $(p=0.03)$.

There was no complication in group A. In group $B, 1$ hip had superficial wound infection and finally healed when oral antibiotics were administered. Two patients complained of greater trochanter consistent mild pain, which recovered at 1 month postoperatively. No lateral femoral cutaneous nerve damage case was reported.

\section{Discussion}

ONFH is a commonly seen disabling condition in the out-patient department, and young individuals are the main sufferers. Cui et al. [23] who conducted a multicentric epidemiologic study based on 6395 ONFH cases found that $\mathrm{ONFH}$ onset mostly at the age between 40 and 50 years. However, the patients enrolled in our study were mainly in their late thirties. It is difficult to discover early-stage ONFH because of concealed morbidity and asymptomatic clinical signs, and it aggravates at a rapid course. Thus, conservative treatment always seems to be not effective. Based on the symptomatic ONFH natural history, the femoral head might be progressively collapsing without operative intervention under that condition. Surgeries that can maintain the femoral head morphology, promote necrotic bone repair, and obtain good hip function, and with no pain are considered as the preferred methods for ONFH management. Many clinical trials have been reported that hippreserving surgeries can slow down the natural history, which aims to prevent femoral head continuous collapse and avoid or delay THA conversion. However, the mosteffective hip-preserving surgeries are still full of controversies and under severe debate [24, 25].

One-stage hip-preserving surgeries own the advantages of faster recovery, shorter hospital stay, and fewer costs than two-stage surgeries [26]. Regardless of the advantages, one-stage bilateral surgeries might also pose greater difficulty on the postoperative rehabilitation, and 


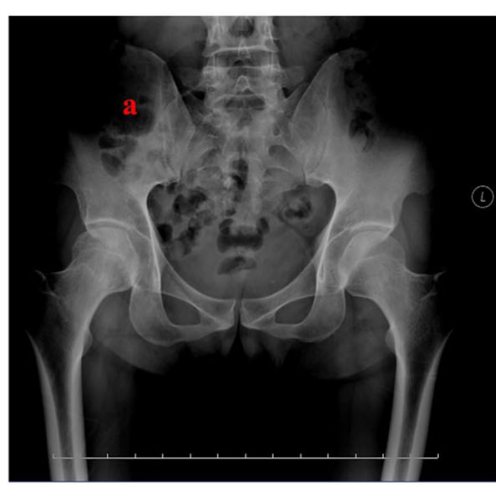

(a)

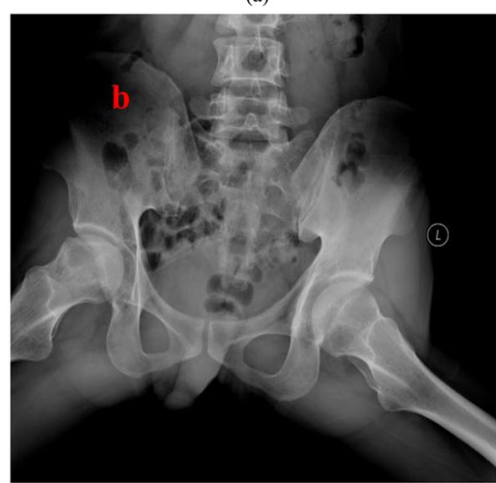

(b)

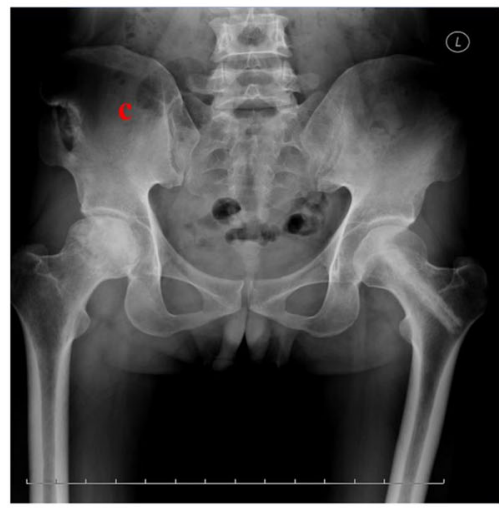

(c)

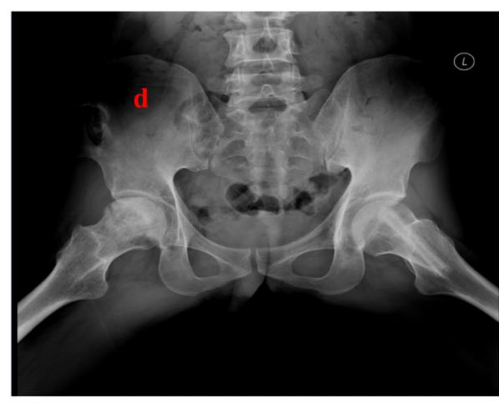

(d)

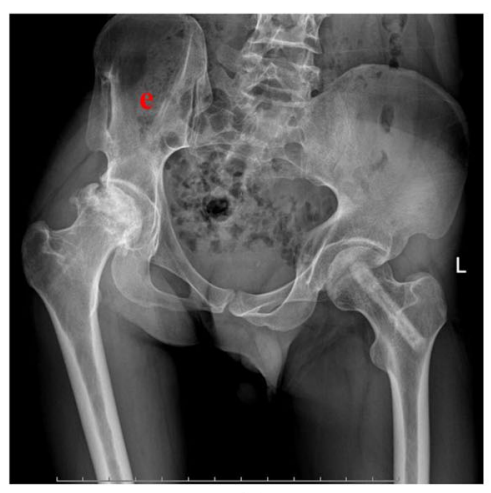

(e)

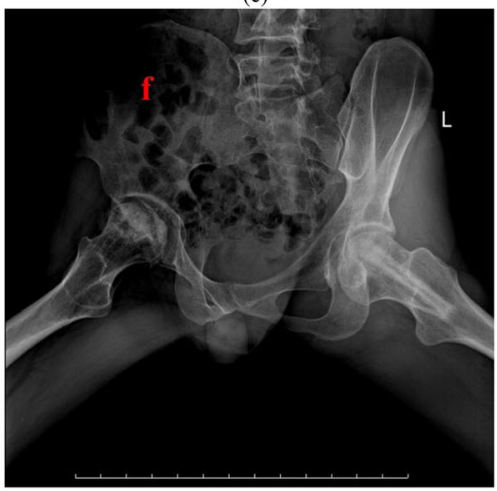

(f)

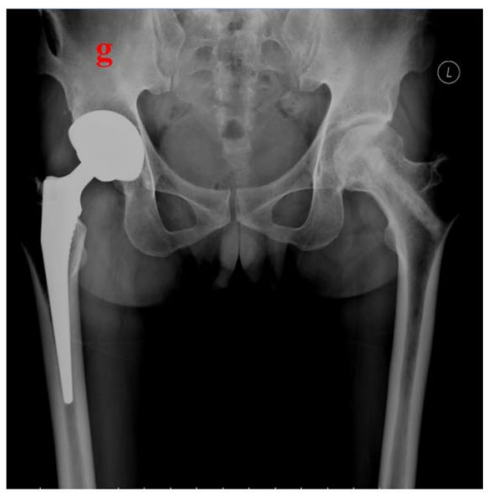

(g)

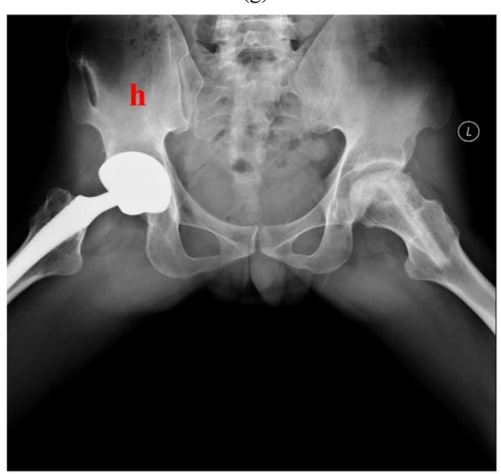

(h)

Fig. 2 (See legend on next page.) 
(See figure on previous page.)

Fig. $\mathbf{2}$ a, b The 38-year-old man was diagnosed with bilateral osteonecrosis of the femoral head due to alcohol overtake. c, d Postoperative 1year X-rays showed both femoral head shape was maintained and the cystic area was reduced or eliminated, which indicates the necrotic repair response was good. e, $\mathbf{f}$ Postoperative 6-year $X$-rays revealed right femoral head collapsed and secondary osteoarthritis occurred, while the left hip was completely healed. $\mathbf{g}, \mathbf{h}$ The patient had a right hip total hip arthroplasty conversion, while the left hip was maintained

longer operation time under analgesic which may increase the risks of complications. However, we deemed that patients benefit more advantages than disadvantages from one-stage hip-preserving surgeries. We have reported that either fibular impaction allografting or vascularized greater trochanter flap autografting can obtain good results [12, 13]. Our results also showed that patients who underwent one-stage abovementioned hippreserving surgeries can achieve great Harris Hip Score improvements and decreased VAS points. Our findings proved that one-stage hip-preserving surgeries could also be effective and safe, which could provide evidencebased complementary methods for the treatment of $\mathrm{ONFH}$. The vascularity of the target vessel is the determining factor for success treatment; thus, we do not recommend traumatic patients to receive vascularized greater trochanter flap combined with autografting because the lateral femoral circumflex artery might be disrupted due to internal fixation of the displaced femoral neck fracture. However, fibular impaction allografting can achieve more pain relief, though no clinical difference was found in terms of MCID. It was probably due to the preoperative higher pain level. From the aspects of femoral head collapse and necrotic repair process, fibular impaction allografting achieved better results than combining bone grafting. It might be attributed to group A mainly having ARCO stage II hips while group B mainly having ARCO stage III hips. It is known to us that necrotic size and femoral head morphology are key factors for prognosis [27]. Besides, the survivorship at a mean 7.0-year follow-up also showed no difference. Our study suggested that the appropriate indication for fibular impaction allografting is ARCO stage II ONFH while ARCO stage III ONFH is the appropriate indication for a combination of vascularized greater trochanter with free iliac bone autografting. We found that the preoperative ARCO stage was slightly negatively correlated with the final outcomes. And alcohol and corticosteroids postoperatively sustain the status as the prognostic risk factors for THA conversion. We need more enrolled cases for further multivariate analysis. Two patients in group B complained donor site consistent mild pain, which might be attributed to the decrease of resistance to tensile stress oriented from surrounding muscles due to the greater trochanter integrity destruction.

There are numerous limitations to the study. Firstly, it was a retrospective case-cohort study. Though the evaluation was made by two independent researchers, the indexed surgeries in both hips were not randomized assigned. It might have option bias, which might affect the outcomes. Further high-level evidence randomized and double-blinded studies are required to confirm the results. Secondly, the number of included patients $(69$ patients with 138 hips) was relatively small. Though selfcontrol study can minimize the impact of specific individuals, however, a large number of cases might provide better convince findings. Besides, we need a larger number of cases to perform a multivariate regression analysis to find other prognostic risk factors, which might help us to make better decisions. Thirdly, the hips in group A were mainly in ARCO II stages, while the affected hips in group B were mainly in ARCO III stages. It might be unfair to evaluate the difference between these two hippreserving surgeries, though no baseline difference was found. Fourthly, the postoperative radiological evaluation was mainly focused on the X-ray images. Postoperative computed tomography $(\mathrm{CT})$ scan and magnetic resonance image (MRI) might be essential to provide a more subjective estimation of the extent of femoral head collapse, repair process, and cartilage status.

\section{Conclusions}

Our study concluded that one-stage fibular impaction allografting and vascularized greater trochanter flap with the combination of cortico-cancellous iliac bone autografting for the management of ONFH could obtain good medium- and long-term outcomes. And fibular impaction allografting showed better radiological results, but no clinical difference was found. We also discovered that the appropriate indication for each procedure was patients with ARCO stages II and III, respectively.

\section{Abbreviations \\ ARCO: Association for Research on Osseous Circulation; BMl: Body mass index; HHS: Harris Hip Score; MCID: Minimal clinically important difference; ONFH: Osteonecrosis of the femoral head; THA: Total hip arthroplasty; VAS: Visual Analogue Scale}

\section{Acknowledgements}

Not applicable.

\section{Authors' contributions}

YZ and WF designed the study. And all surgeries were performed by YZ. WF, $P Y$, and SN completed the draft of the manuscript. LL was responsible for data analysis. PD, JC, JZ, XQ, JL, KJ, HC, ZY, and $H Z$ contributed to data collection and outcomes evaluation. All authors read and approved the final manuscript. 


\section{Funding}

The current study received High-Level Hospital Construction Project of The First Affiliated Hospital of Guangzhou University of Chinese Medicine (Grant number 211010010121).

\section{Availability of data and materials}

All datasets used during the current study are available from the corresponding author on reasonable request.

\section{Ethics approval and consent to participate}

It was approved by the Ethics and Academy Committee of the First Affiliated Hospital of Guangzhou University of Traditional Chinese Medicine ([2017]124).

\section{Consent for publication}

Not applicable.

\section{Competing interests}

The authors declare that they have no competing interests.

\section{Author details}

${ }^{1}$ The First Affiliated Hospital of Guangzhou University of Chinese Medicine, Jichang Road 16\#, District Baiyun, Guangzhou, Guangdong, China. ${ }^{2}$ Guangzhou University of Chinese Medicine, Jichang Road 12\#, District Baiyun, Guangzhou, Guangdong, China. ${ }^{3}$ The First Affiliated Hospital of Guangzhou University of Chinese Medicine, Linnan Medical Research Center of Guangzhou University of Chinese Medicine, Jichang Road 16\#, District Baiyun, Guangzhou, Guangdong, China. ${ }^{4}$ Guangzhou University of Chinese Medicine, Jichang Road 16\#, District Baiyun, Guangzhou, Guangdong, China.

Received: 24 September 2019 Accepted: 8 November 2019 Published online: 21 December 2019

\section{References}

1. Banerjee S, Issa K, Pivec R, Kapadia BH, Khanuja HS, Mont MA. Osteonecrosis of the hip: treatment options and outcomes. Orthop Clin North Am. 2013; 44:463-76.

2. Kang JS, Park S, Song JH, Jung YY, Cho MR, Rhyu KH. Prevalence of osteonecrosis of the femoral head: a nationwide epidemiologic analysis in Korea. J Arthroplast. 2009;1:1178-83.

3. Ficat RP. Idiopathic bone necrosis of the femoral head. Early diagnosis and treatment. J Bone Joint Surg Br. 1985;67:3-9.

4. Hernigou P, Bachir D, Galacteros F. The natural history of symptomatic osteonecrosis in adults with sickle disease. J Bone Joint Surg Am. 2003;85: 500-4.

5. Osawa Y, Seki T, Takegami Y, Kusano T, Makida K, Ishiguro N. Cementless total hip arthroplasty for osteonecrosis and osteoarthritis produce similar results at ten years follow-up when matched for age and gender. Int Orthop. 2018;42(7):1683-8.

6. Lin PC, Wang CJ, Yang KS, Wang FS, Ko JY, Huang CC. Extracorporeal shockwave treatment of the osteonecrosis of the femoral head in systemic lupus erythematosis. J Arthroplast. 2006;21(6):733-59.

7. Disch AC, Matziolis G, Sieve L, Wang P. The treatment of necrosis-associated and idiopathic bone-marrow oedema of the proximal femur by intravenous iloprost. J Bone Joint Surg Br. 2005;87(4):560-4

8. Glueck CJ, Freiberg RA, Sieve L, Wang P. Enoxaparin prevents progression of stages I and II osteonecrosis of the hip. Clin Ortho Relat Re. 2005:435:164-70.

9. Deng P, Zeng J, Li J, Feng W, Chen J, Zeng Y. Screening of serum protein markers for avascular osteonecrosis of femoral head differentially expressed after treatment with Yuanshi Shengmai Chenggu Tablets. Biomed Res Int. 2018;2018:5692735.

10. Wang C, Peng J, Lu S. Summary of the various treatments for osteonecrosis of the femoral head by mechanism: a review. Exp Ther Med. 2014;8(3):700-6.

11. Wang T, Wang W, Yin ZS. Treatment of osteonecrosis of the femoral head with thorough debridement, bone grafting, bone-marrow mononuclear cells implantation. Eur J Orthop Surg Traumatol. 2014;24(2):197-202.

12. Zeng YR, He S, Feng WJ, Li FL, Li J, Jian LY, Zeng JC, Fan YG. Vascularized greater trochanter bone graft, combined free iliac flap and impaction bone grafting for osteonecrosis of the femoral head. Int Orthop. 2013;37(3):391-8.

13. Zeng Y, Qi X, Feng W, Li J, Li F, Zeng J, Yi C, Chen J. One-sided hippreserving and concurrent contralateral total hip arthroplasty for the treatment of bilateral osteonecrosis of the femoral head in different stages: short-medium term outcomes. BMC Musculoskelet Disord. 2015;16:133.

14. Song WS, Yoo JJ, Kim HJ. Results of multiple drilling compared with those of conventional methods of core decompression. Clin Orthop Relat Res. 2007:454:139-46

15. Sugioka Y, Hotokebuchi T, Tsutsui H. Transtrochanteric anterior rotational osteotomy for idiopathic and steroid-induced necrosis of the femoral head. Indications and long-term results. Clin Orthop Relat Res. 1992;277:111-20.

16. Song H, Tao L, Wang F, Wang W, Wei Y, Shen W, Zhou F. Effect of bone mesenchymal stem cells transplantation on the micro-environment of early osteonecrosis of the femoral head. Int J Clin Exp Pathol. 2015;8(11):14528-34.

17. Sugano N, Takaoka K, Ohzono K, Matsui M, Masuhara K, Ono K. Prognostication of nontraumatic avascular necrosis of the femoral head: significance of location and size of necrotic lesion. Clin Orthop Relat Res. 1994:303:155-64.

18. Lee MS, Chang YH, Chao EK, Shih $\mathrm{CH}$. Conditions before collapse of the contralateral hip in osteonecrosis of the femoral head. Chang Gung Med J. 2002;25(4):228-37.

19. Marcus ND, Enneking WF, Massam RA. The silent hip in idiopathic aseptic necrosis: treatment by bone-grafting. J Bone Joint Surg Am. 1973;55(7): 1351-66.

20. Shih L-Y, Wong Y-C, Shih H-N. One-stage hip arthroplasty and bone grafting for bilateral femoral head osteonecrosis. Clin Orthop Relat Res. 2009;67: 1522-8.

21. Cao L, Guo C, Chen J, Chen Z, Yan Z. Free vascularized fibular grafting improves vascularity compared with core decompression in femoral head osteonecrosis: a randomized clinical trial. Clin Orthop Relat Res. 2017;475: 2230-40.

22. Jasvinder A, Singh CS, Harmsen S, Lewallen D. Clinically important improvement thresholds for Harris Hip Score and its ability to predict revision risk after primary total hip arthroplasty. BMC Musculoskelet Disord. 2016;17:256.

23. Cui L, Zhuang Q, Lin J, Jin J, Zhang K, Cao L, Lin J, Yan S, Guo W, He W, Pei F, Zhou $Y$, Weng X. Multicentric epidemiologic study on six thousand three hundred and ninety five cases of femoral head osteonecrosis in China. Int Orthop. 2016;40:267-76.

24. Korompilias AV, Beris AE, Lykissas MG, Kostas-Agnantis IP, Soucacos PN. Femoral head osteonecrosis: why choose free vascularized fubular grafting. Microsurgery. 2011;31:223-8.

25. Chen $\mathrm{CH}$, Chang JK, Lai K, Hou SM, Chang CH, Wang GJ. Alendronate in the prevention of collapse of the femoral head in nontraumatic osteonecrosis: a two-year multicenter, prospective, randomized, double-blinded, placebocontrolled study. Arthritis Rheum. 2012;64:1572-8.

26. Jergesen HE, Poss R, Sledge CB. Bilateral total hip and knee replacement in adults with rheumatoid arthritis: an evaluation of function. Clin Orthop Relat Res. 1978:137:120-8.

27. Tabatabaee RM, Saberi S, Parvizi J, Mortazavi SM, Farzan M. Combining concentrated autologous bone marrow stem cells injection with core decompression improves outcomes for patients with early-stage osteonecrosis of the femoral head: a comparative study. J Arthroplast. 2015: 30(9 suppl):11-5.

\section{Publisher's Note}

Springer Nature remains neutral with regard to jurisdictional claims in published maps and institutional affiliations.

Ready to submit your research? Choose BMC and benefit from:

- fast, convenient online submission

- thorough peer review by experienced researchers in your field

- rapid publication on acceptance

- support for research data, including large and complex data types

- gold Open Access which fosters wider collaboration and increased citations

- maximum visibility for your research: over $100 \mathrm{M}$ website views per year

At $\mathrm{BMC}$, research is always in progress.

Learn more biomedcentral.com/submission 